\section{Palmitate is not a TLR4 agonist}

Based on previous evidence, it was thought that Toll-like receptor 4 (TLR4) sensed fatty acids to induce inflammation and metabolic dysfunction in obesity. Now, Graeme Lancaster, Mark Febbraio and colleagues have shed new light on this hypothesis, showing that TLR4 is not a receptor for palmitate, a long-chain saturated fatty acid, in vivo.

"From the mid-to-late 2000s, numerous in vitro and in vivo studies confirmed a major role for TLR4 in lipid-induced inflammation, with the idea being that saturated fatty acids were ligands for TLR4," explains Lancaster. "However, there are several lines of evidence that argue against saturated fatty acids being direct agonists of TLR4 and that is why we began to explore this area a number of years ago."

In the present study, Lancaster, Febbraio and colleagues used a number of different approaches to investigate whether palmitate, which has been used previously by investigators to induce cellular inflammation, was an agonist of TLR4. The authors first assessed whether TLR4 undergoes dimerization and endocytosis in the presence of palmitate. They then used a computational technique called molecular dynamics simulations to assess whether palmitate acted in a similar manner to lipopolysaccharide, which is the canonical TLR4 agonist.

The authors report that they were unable to find any evidence that palmitate is a ligand of TLR4. "While our study might appear to fly in the face of over a decade's worth of previous work, it doesn't," adds Febbraio. “We propose that certain signals, for example, obesity-induced increases in circulating concentrations of lipopolysaccharide or perhaps endogenous TLR4 ligands emanating from obese adipose tissue, activate adipose tissue-resident macrophages, stimulating them to adopt the classically-activated, or M1, phenotype." This TLR4-dependent reprogramming of macrophage metabolism renders the adipose tissue-resident macrophages particularly sensitive to subsequent stimulation with long-chain saturated fatty acids, which induce further

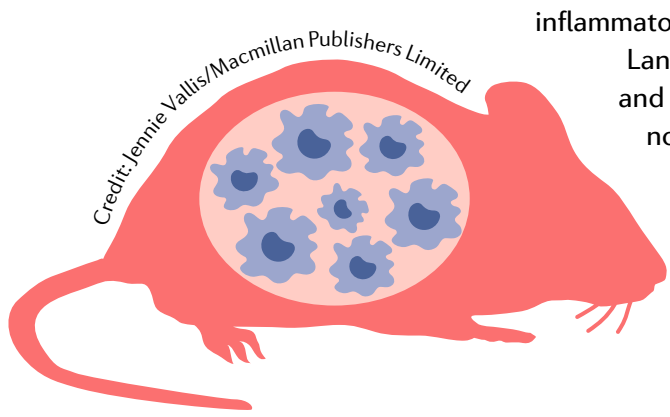
Lancaster, Febbraio and colleagues are now working on trying to identify the receptors that mediate the uptake of fatty acids into macrophages.

Alan Morris

ORIGINAL ARTICLE Lancaster, G. I. et al. Evidence that TLR4 is not a receptor for saturated fatty acids but mediates lipid-induced inflammation by reprogramming macrophage metabolism. Cell Metab. https://doi.org/10.1016/j. cmet.2018.03.014 (2018)

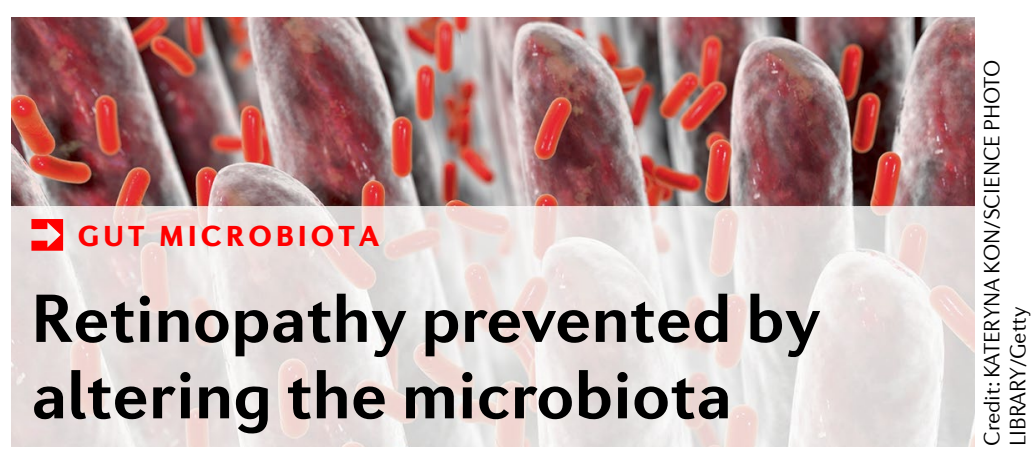

Changes to caloric intake, such as chronic calorie restriction and intermittent fasting, are known to protect against the development of metabolic diseases. However, how these regimens might affect the complications of metabolic diseases has been unclear. Now, new research demonstrates that intermittent fasting can prevent the development of diabetic retinopathy in mice by altering the composition of the gut microbiota.

The researchers used four sets of mice: diabetic (db/db) mice and control mice that were fed either ad libitum or with intermittent fasting (with fasting every other day) for 7 months. The fasting regime did not alter levels of $\mathrm{HbA}_{1 \mathrm{c}}$, but did lead to increased survival of the fasted $\mathrm{db} / \mathrm{db}$ mice compared with the diabetic mice fed ad libitum.

Next, the researchers assessed the number of acellular retinal capillaries (an early marker of diabetic retinopathy). As would be expected, the $\mathrm{db} / \mathrm{db}$ mice fed ad libitum had increased numbers of acellular retinal capillaries compared with the control mice fed ad libitum. However, no such increase was seen in the $\mathrm{db} / \mathrm{db}$ mice on the intermittent fasting regimen. The fasting regimen also limited the infiltration of inflammatory cells into the retina, which is another marker of diabetic retinopathy.

Given that the microbiome can influence the progression of diabetes mellitus, the authors hypothesized that the beneficial effects of intermittent fasting could be the result of changes in the microbiome. By analysing faecal samples, they were able to show that $\mathrm{db} / \mathrm{db}$ mice on the fasting regimen had increased levels of Firmicutes and reduced levels of Bacteroidetes and Verrucomicrobia. These changes in the microbiota were reflected in altered bile acid metabolism in the fasted diabetic mice. For instance, levels of the bile acid product taurochenodeoxycholate (which is converted to the neuroprotective tauroursodeoxycholate (TUDCA)) were increased in the fasted mice.

To investigate whether there was a mechanistic link between diabetic retinopathy and the changes in bile acid metabolism induced by the altered microbiota composition, the researchers examined the expression of G protein-coupled bile acid receptor 1 (GPBAR1; also known as TGR5, the receptor for TUDCA). Intermittent fasting did not alter expression levels of TGR5 in the neural retinal cells of the mice; however, retinal levels of tumour necrosis factor mRNA (which is a downstream target of TGR5 activation) were reduced in fasted mice. In a second mouse model, the researchers administered INT-767, which activates TGR5. Untreated mice developed diabetic retinopathy, but the treated mice did not. "We showed that the effect of intermittent fasting was through changes in the gut microbiota that generated increased levels of bacteria that transform primary bile acids to the particularly potent secondary bile acid, TUDCA," explains author Maria Grant. "TUDCA enters the circulation and can activate protective receptors (TGR5) in the retina, in particular in the neural cells."

The authors suggest that intermittent fasting could be used therapeutically to increase levels of TUDCA in patients, thus potentially protecting against diabetic retinopathy. TGR5 could also be a new therapeutic target for diabetic retinopathy.

Claire Greenhill

ORIGINAL ARTICLE Beli, E. et al. Restructuring of the gut microbiota by intermittent fasting prevents retinopathy and prolongs survival in $\mathrm{db} / \mathrm{db}$ mice. Diabetes https://doi.org/10.2337/ db18-0158 (2018) 